\title{
Robotic Assembly System for Steel Structures
}

\author{
Ci-Jyun Liang ${ }^{1,2}$ and Shih-Chung Kang ${ }^{1,2^{*}}$ \\ ${ }^{1}$ Department of Civil Engineering, National Taiwan University \\ ${ }^{2}$ No.188, Sec. 3, Xinhai Rd., Da'an Dist., Taipei City 106, Taiwan (R.O.C.) \\ *Corresponding author's e-mail: sckang@ntu.edu.tw
}

\begin{abstract}
Workers are required to stand on dangerous unfinished steel structures to assemble elements manually. Therefore, we developed a robotic assembly system (RAS) to prevent accidental falls. The RAS consists of four methods: rotation, alignment, bolting, and unloading. The rotation method utilizes a flywheel equipped on top of a rigging beam to rotate the beam. The vertical alignment relies on a camera and a marker to align the altitude of the beam. The horizontal alignment relies on a specially designed shape that can smoothly guide the beam to the right position. The bolting method adds an additional plug hole above each bolt hole to assemble the beam. The unloading method uses pin mechanisms and motors to unload the cable and the RAS. The system is tested in a scaled indoor experiment and the results show that the process is finished without workers stay in the high place. In conclusion, the RAS helps reduce accidental falls, is suitable to the current erection method, and can be broadly introduced to existing sites.
\end{abstract}

\section{KEYWORDS}

steel beam assembly, construction automation, construction safety, auto joint, rotation method

\section{INTRODUCTION}

The steel beam assembly process is a crucial procedure in large high-rise steel structure construction projects; however, it relies heavily on manual labor and time consuming (Chi and Kang 2010). During the process, workers are required to stand on a narrow steel beam in a high-rise location with only a simple safety cable. Accidental falls sometimes occur and cause serious injuries, which is the main type of accident that occurred at construction site (OSHA 2013). Therefore, removing human workers from high place is the primary goal to improve construction safety. A GYAPTS (Wakisaka et al. 2000) device was implemented in an automatic construction system and used in a reinforced concrete building site. A Stewart platform crane and the ATLSS connection can provide a three-dimensional fully controllable manipulator (Vincent Viscomi et al. 1994). The ATLSS connection is a joint that allows fast 
and easy assembly for steel frames. A scissor jack-type manipulator combined with a "construction factory" constructed automatic steel beam assembly has been developed (Jung et al. 2008). The construction factory is a large workspace, which is constructed outside the building and will move vertically during the construction process (Kim et al. 2009). The scissor jack-type manipulator can move in the construction factory and lifts the steel beam to its assembly location. A rail-boom combined robotic system and wire-suspended position system were also introduced to achieve an automatic steel beam assembly (Hyungdo et al. 2007). A bolting robot includes a bolting tool and a camera (Jung et al. 2013). Image processing was implemented to detect the bolt hole (Baek et al. 2011). A bolting mechanism was developed to assemble the bolts (Chu et al. 2013). The bolting robot was attached to the construction factory and the user can control it remotely (Jung et al. 2013; Jung et al. 2013). In this study, we develop a robotic assembly system (RAS) for steel structure, which aims to improve the safety of the steel beam assembly process. The RAS can rotate, align, bolt, and assemble a beam without requiring steel workers to be in high locations. Comparing to the previous study, this system is easy-removable and light-weight. The RAS fits current erection method and can be broadly introduced to existing construction sites.

\section{THE ROBOTIC ASSEMBLY SYSTEM}

A detailed procedure of erection and assembly with the RAS is illustrated in Figure 1. Two workers are required to operate the system; one who is a ground operator and the other who is a tower crane operator. First, the ground operator attaches the steel beam to the tower crane hook and prepares it for erection. Second, the tower crane operator transports the beam to the target position, which is the top of the assembly position, and aligns it roughly. Third, the RAS assists the operator to adjust the height of the beam to a proper level, which enables the beam to be successfully connected to the column later. Fourth, the RAS rotates the beam to the correct angle, which enables the steel beam to be connected to the column. Fifth, the crane operator adjusts the horizontal position of the beam accurately using the RAS. Notice that if the beam failed to reach the correct position, it has to go back to the rotation step and repeat the process. Sixth, the beam is assembled with bolts, after which the temporary connection is completed. The ground operators must check whether all bolts have been assembled. The process returns to the rough alignment step if it has failed to assemble all the bolts. Seventh, the RAS unloads the beam-hook connecting cable. Eighth, the tower crane removes the RAS and repositions for the next beam. The RAS consists of four key methods, namely the rotation, alignment, bolting, and unloading methods. A detailed description of these methods is given in the proceeding sections. 




Figure 1 The procedure of the beam erection and assembly with the RAS.

\section{Rotation Method}

We employ the principle of conservation of angular momentum to achieve the rotation method. A rotation box with a flywheel is equipped on top of the rigging beam, as shown in Figure 2, to generate angular momentum and the beam will generate an inverse angular momentum. We assume the friction between the hook and the block is zero. We also neglect the effect of wind due to the massive weight of the rigging beam. The angular velocity of the beam given by the conservation of angular momentum equation is expressed as follows:

$$
\omega_{b}=\frac{I_{w}}{I_{b}} \omega_{w}
$$

where $I_{w}, I_{b}$ represent the moment of inertia of the flywheel and the rigged beam, respectively; and $\omega_{w}, \omega_{b}$ represent the angular velocity of the flywheel and the rigged beam, respectively. The angular velocity of the flywheel is provided by a motor inside the rotation box, which is also the maximum revolution per minute of the motor $\omega_{m}$. The angular velocity of the flywheel has two different periods and each has different description. First is the accelerating period and second is the constant velocity period. In the accelerating period, we assume a constant acceleration $\alpha$ during the process. The angular velocity is $\omega_{w}=\alpha t_{a}$, where $t_{a}$ is the accelerating time to reach the maximum revolution per minute $\omega_{m}$. In the constant velocity period, we assume the angular velocity of the motor always reaches the maximum revolution per time. The angular velocity is equal to the maximum revolution per time. Therefore, the angular velocity of the rigged beam can be derived from (1) and the angular velocity of the flywheel. 


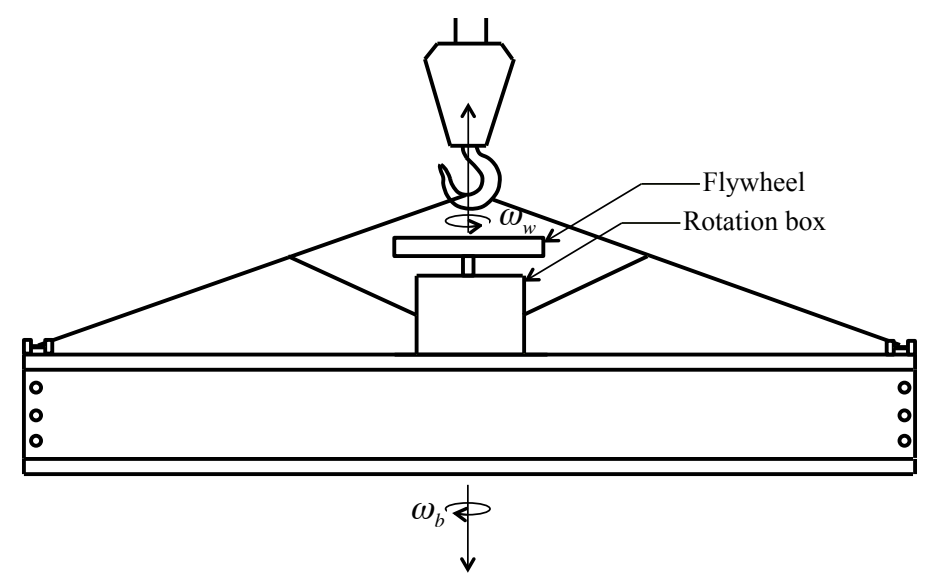

Figure 2 The mathematical model of the rotation method (side view).

\section{Alignment Method}

The alignment method is divided into two parts, namely vertical alignment and horizontal alignment. The objective of vertical alignment is to check whether the rigging beam reaches the correct height. We use a camera to capture the marker on the column and a light signal to inform the ground operator whether the beam reaches the correct height. Figure 3 illustrates the vertical alignment method. In order to assemble the beam, the vertical alignment position of the rigging beam must be slightly higher than the corbel. Length $d$ represents the distance from the center of the camera lens to the top surface of the beam. Length $\delta$ is the vertical distance between the beam and the corbel, which is also the distance from the center of the plug hole to the bolt hole. Therefore, the centroid of the marker is $d+\delta$ higher from the corbel. Length $L$ is the distance from the center of the camera lens to the column, which we will use to determine the marker size. The marker will be printed on the column before assembly process and the size of it is determined by erection swag. Figure 4 shows the mathematical model of the influence of erection swag on the marker size. The marker length $\Delta$ is expressed as follows:

$$
\Delta=2 L \tan \theta
$$



Figure 3 (a)The vertical alignment method (partial side view) and (b)the bolting steel plate (front view). 
where $L$ represents the distance between the camera and the column and $\theta$ represents the pendulum angle. The pendulum equation, according Kuo and Kang (2014), is expressed as follows:

$$
\frac{d^{2} \theta}{d t^{2}}=\frac{a}{l} \cos \theta-\frac{g}{l} \sin \theta
$$

where $a$ represents the crane operation acceleration, $l$ represents the crane cable length, and $g$ represents gravity. The marker width $B$ is expressed as follows:

$$
B=2 l \sin \theta
$$

Therefore, we can determine the marker length $\Delta$ and width $B$ with (2), (3) and (4).


Figure 4 The mathematical model of the influence to the marker size due to the erection swag: (a)left side view and (b)back view.

The objective of the horizontal alignment is to adjust the rigging beam to the assigned position. We change the shape of the flange plates into parallelograms to avoid the beam being stuck during the rotation process, as shown in Figure 5. In addition, in case the beam is not at the correct position it can be easily moved by the tower crane due to its shape.

(a)
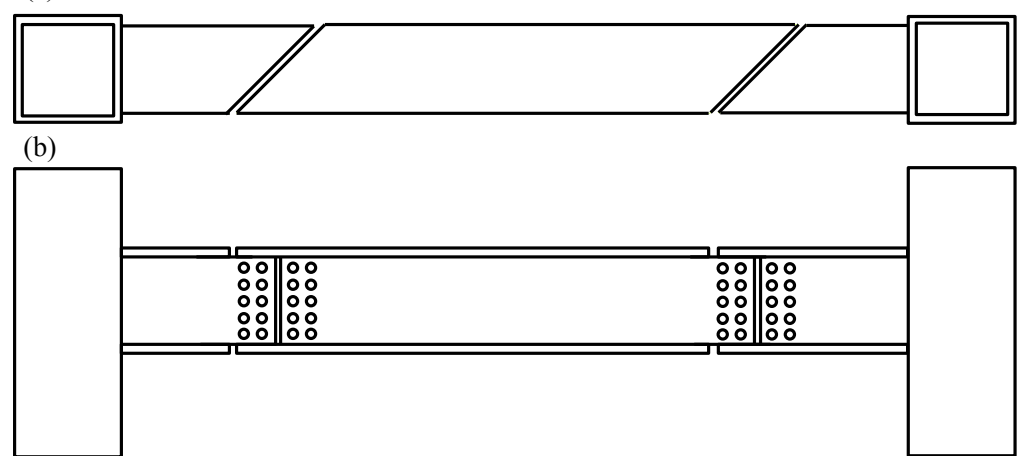

Figure 5 The horizontal alignment method: (a)top view and (b)side view 


\section{Bolting and unloading Method}

For providing a faster bolting process, we use the "plug and play" method. Figure 3(b) shows the front view of the bolting steel plate. We add two additional plug holes through the bolt holes as only two bolts are needed for a temporary connection. After finishing the horizontal alignment, the bolts have been plugged into the plug holes. The crane operator then releases the rigged beam and the bolts will slide into the bolt holes, as shown in Figure 6, and the bolts assembly step is completed. The unloading method is used to remove the RAS and unload the cable. We use a pin mechanism, cable, and motor to perform the unloading method. After the bolting assembly step is completed, the motor starts to roll the cable and extract the pin from the mechanism. The RAS is then unloaded from the rigged beam and can reposition for the next target.



(b) (c)





Section A-A (d)



Figure 6 The concept of the bolting method: (a)(c)before releasing and (b)(d)after releasing.

\section{SCALED INDOOR EXPERIMENT}

For validating the RAS, we implement a scaled indoor experiment, which includes a tower crane and a steel structure. We used KUKA KR 16 CR to simulate the tower crane, block boards and angle steels to build the steel structure. The steel structure is an experimental structure from the National Center for Research on Earthquake Engineering (Lin et al. 2013). The rotation box was demonstrated by the TETRIX ${ }^{\circledR}$ and LEGO ${ }^{\circledR}$ Mindstorms NXT. The LabVIEW was used to program the controlling software. For the vertical alignment, we used the GHI .Net Gadgeteer kit camera module, LED module and green paint.

\section{Result}

The procedure to achieve results follows the process of beam erection and assembly with the RAS, as shown in Figure 1. The camera on the rotation box is rotated to the column orientation and the image is captured, as shown in Figure 7(a). We use image processing to detect the color in the center of the image. If the camera detects a green color, the LED light will start to flash, as shown in Figure 7(b). The rotation time of the proposed system is illustrated in Table 1. Beam I is the real size of the experimental structure (Lin et al. 2013) 
and Beam II is a steel beam from a real steel structure. We use a motor with $1500 \mathrm{rpm}$ and a $1500 \mathrm{~kg}-\mathrm{m}^{2}$ flywheel for the real rotation box. The accelerating time $t_{a}$ is 10 seconds. The angle of rotation is set as 90 degrees.


Figure 7 The vertical alignment: (a)adjust the altitude and (b)achieve the vertical alignment.

Table 1 The comparison of the rotation time.

\begin{tabular}{cccc}
\hline & Beam I & Beam II & Scaled Beam \\
\hline Beam section $(\mathrm{mm})$ & $\mathrm{H} 800 \times 400 \times 22 \times 32$ & $\mathrm{H} 800 \times 300 \times 14 \times 26$ & $\mathrm{H} 290 \times 160 \times 15 \times 15$ \\
\hline $\mathrm{L}(\mathrm{mm})$ & 5,000 & 10,000 & 1,720 \\
\hline Weight $(\mathrm{kg})$ & 1,630 & 2,099 & 5 \\
\hline$I_{b}\left(\mathrm{~kg}-\mathrm{m}^{2}\right)$ & 3,396 & 17,492 & 1.989 \\
\hline$\omega_{m}(\mathrm{rpm})$ & 1,500 & 1,500 & 152 \\
\hline$I_{w}\left(\mathrm{~kg}-\mathrm{m}^{2}\right)$ & 2 & 2 & $8.982 \times 10^{-3}$ \\
\hline$\omega_{b}(\mathrm{deg} / \mathrm{s})$ & 5.298 & 1.032 & 7.717 \\
\hline$t_{a}(\mathrm{sec})$ & 10 & 10 & $7.905 \times 10^{-2}$ \\
\hline Rotation time $(\mathrm{sec})$ & $\mathbf{2 1 . 9 9}$ & $\mathbf{9 2 . 2 1}$ & $\mathbf{1 1 . 7 4}$ \\
\hline
\end{tabular}

\section{CONCLUSION}

We developed a robotic beam assembly system (RAS) for a steel structure. The rotation, alignment, bolting, and unloading methods are the four main parts of the RAS. The rotation method utilizes a flywheel to rotate the beam. The alignment method utilizes a camera and a marker on the column to ensure correct altitude of the beam. By using a parallelogram flange plate, the beam can be easily aligned. For bolting, we add an additional plug hole above the bolt hole; therefore, the bolt can plug and slide into the bolt hole. The unloading method employs a pin mechanism to unload. A scaled indoor experiment was implemented to verify the system. We found out that the RAS is able to assemble the beam without human workers standing in the high place. In conclusion, the RAS is intended to replace human workers in high-rise buildings. This could reduce accidental falls of the steel beam assembly process. 


\section{REFERENCES}

Baek, G. R., Mo, Y. H., Jeong, J. S., Park, J. M., and Lim, M. T. "Cognition system of bolt hole using template matching." Proc., International Symposium on Automation and Robotics in Construction, 685-688.

Chi, H.-L., and Kang, S.-C. (2010). "A physics-based simulation approach for cooperative erection activities." Automation in Construction, 19(6), 750-761.

Chu, B., Jung, K., Lim, M.-T., and Hong, D. (2013). "Robot-based construction automation: An application to steel beam assembly (Part I)." Automation in Construction, 32, 46-61.

Hyungdo, N., Wooseok, C., Dongseok, R., YongKwun, L., Se-Han, L., and Bumjae, R. "Design of a bolting robot for constructing steel structure." Proc., International Conference on Control, Automation and Systems, 1946-1949.

Jung, K., Chu, B., Bae, K., Lee, Y., Hong, D., Park, S., and Lim, M.-T. "Development of automation system for steel construction based on robotic crane." Proc., International Conference on Smart Manufacturing Application, IEEE, Kintex, Gyeonggi-do, Korea, 486-489.

Jung, K., Chu, B., and Hong, D. (2013). "Robot-based construction automation: An application to steel beam assembly (Part II)." Automation in Construction, 32, 62-79.

Jung, K., Chu, B., Park, S., and Hong, D. (2013). "An implementation of a teleoperation system for robotic beam assembly in construction." Int. J. Precis. Eng. Manuf., 14(3), 351-358.

Kim, D. W., An, S. H., Cho, H., Jeong, J. W., Lee, B. H., Doh, N. L., and Kang, K. I. (2009). "Development of conceptual model of construction factory for automated construction." Build Environ, 44(8), 1634-1642.

Kuo, T.-Y. T., and Kang, S.-C. J. (2014). "Control of fast crane operation." Automation in Construction, 42, 25-35.

Lin, K.-C., Jhuang, S.-J., and Lin, C.-H. (2013). "Study on Seismic Performance of Steel Shop-Fabricated Moment Connections." National Center for Research on Earthquake Engineering.

OSHA (2013). "Construction's "Fatal Four"." $<$ https://www.osha.gov/oshstats/commonstats.html\%3E. (Mar. 10, 2015).

Vincent Viscomi, B., Michalerya, W. D., and Lu, L.-W. (1994). "Automated construction in the ATLSS integrated building systems." Automation in Construction, 3(1), 35-43.

Wakisaka, T., Furuya, N., Inoue, Y., and Shiokawa, T. (2000). "Automated construction system for high-rise reinforced concrete buildings." Automation in Construction, 9(3), 229-250. 